\title{
Introduction to Carbon Nanotube Hybrid Textiles
}

\author{
Devika Chauhan, Chenhao Xu, Daniel Chen, Ashley Kubley, Brooke Brandewie, Guangfeng Hou, Weifeng \\ Li, Vianessa Ng, Massoud Rabiee, Marc Cahay, Woo Kyun Kim, Sumeet Chaudhary, Khwaja Moinuddin, \\ Michael Paine, Richard Kleismit, David Mast, Surendra Devarakonda, Sang Young Son, Vesselin N Shanov \\ and Mark J Schulz*
}

University of Cincinnati, USA

*Corresponding author: Mark J Schulz, Professor and Co Director of Nanoworld

Received Date: November 27, 2018

Laboratories, University of Cincinnati, USA.

Published Date: January 30, 2019

\begin{abstract}
Carbon nanotube hybrid textiles (CNHT) are produced from a carbon nanotube (CNT) gas phase pyrolysis floating catalyst process with particle injection. The CNT based metal and ceramic textiles produced can be customized for different applications. Research is underway by a large group of students and faculty members working in different disciplines to further investigate injection of different types of nanoparticles in order to wet the nanotubes and to customize the properties of textiles. A brief introduction to this new material is given in this paper.
\end{abstract}

\section{Overview of Carbon Nanotube Hybrid Textiles}

Synthesis of Carbon Nanotube Hybrid Textiles (CNHT) is based on the in-situ hybridization of carbon nanotubes. CNHT represent a new capability in the field of textiles [1]. The significance is that CNHT technology can produce carbon-metallic, carbon-ceramic or various carbon-compound sheet and yarn materials customized for textile and apparel applications. This overall research area is emerging and will bring a new material system to industry. Multi-functionality (strength, toughness, electrical and thermal conductivity) might be designed into carbon hybrid materials (fabric and yarn) for applications including technical textiles, fashion apparel, composites, structures, electrical conductors, low density structures, and other applications. Industrialization of CNHT will affect many industries and provide economic benefits and jobs. This high rate manufacturing at a competitive cost depends on improving and customizing the properties of CNHT. This paper describes initial research performed to synthesize CNHT.

CNHT are formed by injecting nano or micro particles (NMP) into the gas phase pyrolysis method. The method is under development (patent pending, [2]) and involves CNT synthesis with particle injection technology added. The advantage of the new process is that metals and ceramics and various compound nanoparticles can be integrated into the nanotube synthesis process to produce sheet to be used in textile manufacturing. The carbon hybrid textile formed can be customized for specific applications. CNHT is a onestep process in which the integrated NMP can be physically and or chemically linked to the CNT sock and resulting sheet. The one-step process simplifies manufacturing as compared to other approaches such as electroplating that add metals to carbon nanotubes. The down side is that CNHT manufacturing is a complex process, and possibly more expensive than other techniques depending on the type of particle used. CNHT manufacturing also has additional environmental health and safety considerations compared to pristine CNT manufacturing. Special safety procedures related to protecting against the possible toxicity and flammability of the particles are needed when injecting NMP. One goal of developing the CNHT process is to bond together nanotube bundles to increase the properties (strength and conductivity) of nanotube sheet and textiles. Initial results from developing the CNHT manufacturing technique are related to producing textiles are presented in this paper.

\section{Manufacturing CNHT Textiles}

CNT sheet is being developed around the world to form advanced lightweight materials [3-7]. The material cost is high, and the manufacturing throughput is low. Alternatively, metals have different properties including low cost and high conductivities, while ceramics provide abrasion resistance and fire retardancy. A barrier in materials science has been the inability to manufacture a material that integrates carbon materials and metals or ceramics together to form a reliable material whose properties can be customized to meet specific application requirements. Much 
research has been focused on developing nanocomposite materials for structural applications or electrical conduction. An alternative application is to produce nanotube hybrid sheet for use as performance fabrics and textiles. Shown here are early results from developing a continuous manufacturing process to integrate NMP with CNT to produce continuous textile material whose properties vary based on the constituent metals. A difference is the CNHT produced here is a nonwoven material whereas most textiles are woven using fibers.

CNHT are formed using a modified floating catalyst gas phase pyrolysis method that produces carbon nanotubes (CNT). Carbon Metals may be formed by joining carbon atoms or CNT with metal/ alloy atoms or clusters of atoms in a high temperature reaction. The metal atoms are supplied as metal NMP in the high temperature nanotube floating catalyst synthesis process. The CNT and metals join chemically or physically to produce a nanotube sock (Figure 1) that is wound on a drum. The CNHT process allows the properties of sheet to be customized to a limited degree based on the NMP used, and the end application goal. Conductivity, strength, porosity, capillarity, flame retardancy, and other properties can be partially tailored based on the NMP material, size, and geometry. A key advance in the process is the rapid integration of the precursor metals at high temperature, the short growth time, and fast cooling of the carbon species. Fast windup of the nanotube sock produces partially aligned tape. The CNT and metals interlock physically or chemically to form a semi-homogeneous material. Many combinations of materials design are possible. CNHT is a new perspective in manufacturing and can provide new materials for engineered systems.

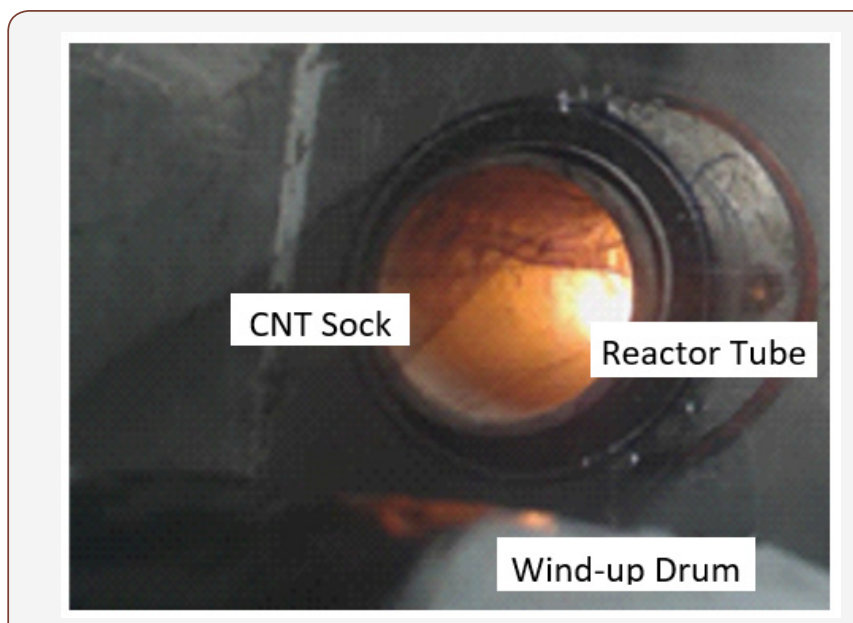

Figure 1: Winding CNT sock to form fabric.

Figure 2 shows SEM images of CNT sheet. Figure 2a, b are the pristine samples where the particles are iron catalyst particles and are a normal impurity of the process. Figure 2c, $d$ are samples with injecting silver coated copper platelets which are larger particles than the iron catalyst.

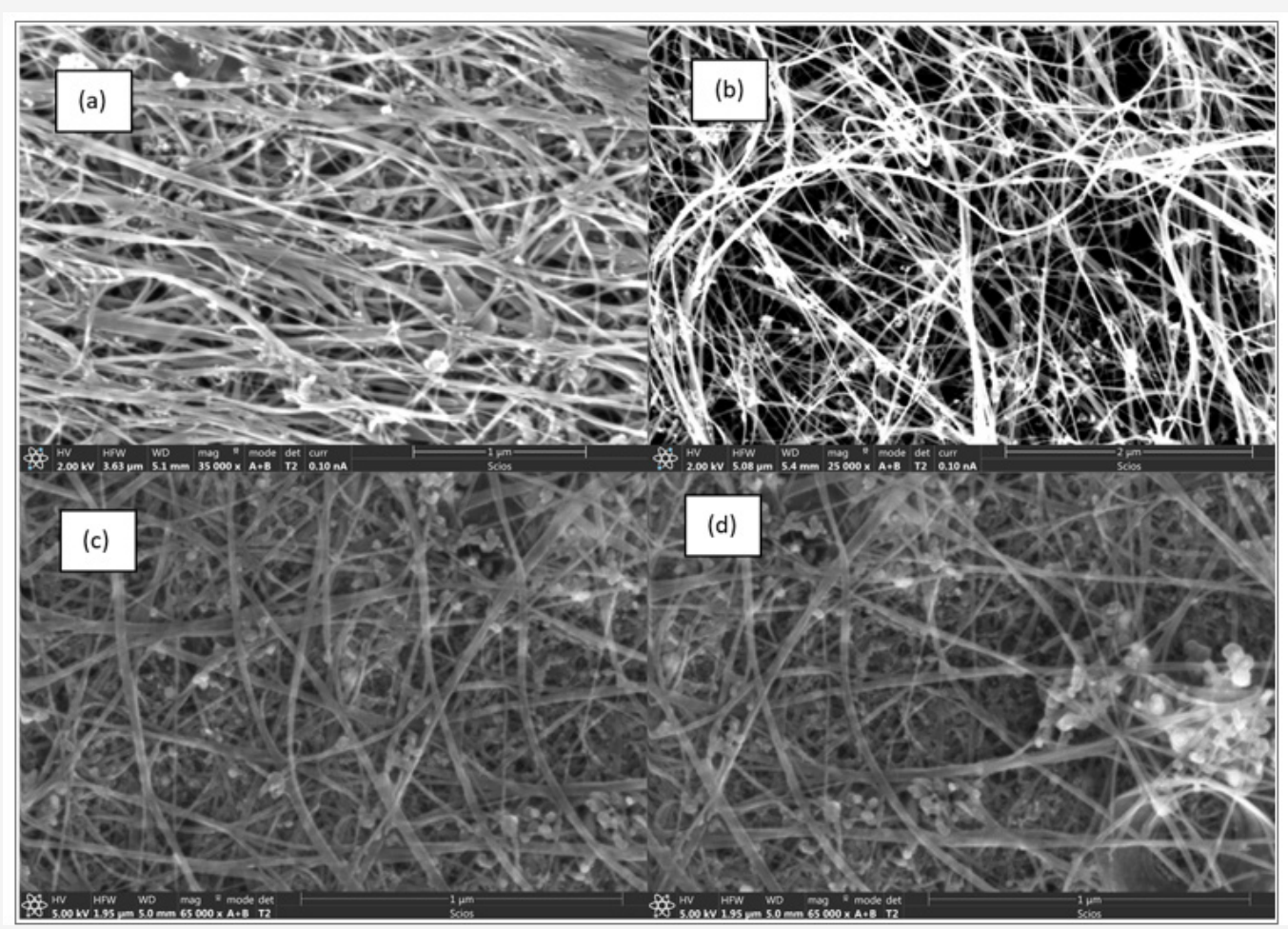

Figure 2: SEM images of CNT sheet. (a, b) are pristine CNT samples, the particles are iron catalyst, a normal impurity of the process. Sample $a$ is collected at 30 RPM, sample $b$ at 20 RPM speed of the wind-up drum. Faster winding aligns more of the nanotube bundles in one direction; (c, d) Samples are CNT-silver coated copper hybrid samples. The samples are not fully densified.

Figure 3 shows a sample with silver coated copper NMP. These samples contain a small percentage of Ag-coated $\mathrm{Cu}$ particles. The NMP are expected to improve the strength, electrical and thermal conductivity of the sheet. However, higher loading of smaller particles is needed to provide a large improvement in properties. CNHT properties can be customized based on the type of particles used. This suggests the possibility of producing "designer textiles" that will have application specific properties. 


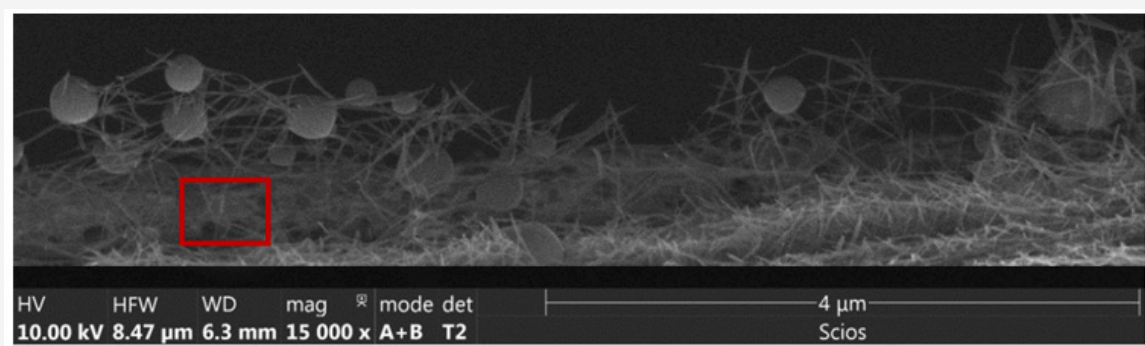

Figure 3: CNT-Ag-Cu hybrid material with metal particles on the surface. Metal particles may "glue" bundles of nanotubes together if the metal can wet the nanotubes. The gluing may improve the electrical conductivity of the sheet and also increase the mechanical stiffness and strength of the fabric.

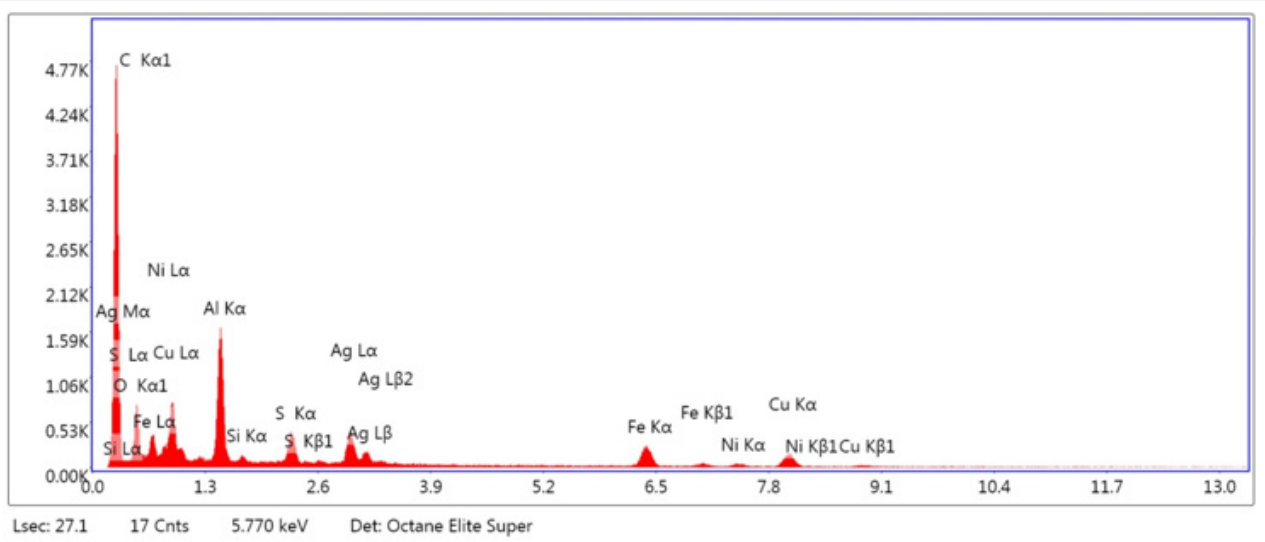

Figure 4: EDAX results from the CNT sheet with Ag-Cu particles.

X-Ray analysis (EDAX) results from one spot shown with red square on the sample in Figure 3 are shown in Figure 4. Corresponding eZAF Smart Quant Results are shown in Table 1. The concentrations of chemicals vary for different positions on the textile. This structural and chemical characterization verifies the carbon-metal composition of the textile material.

Table 1 results quantify the chemical composition of the CNHT. The weight percentage of an element is the weight of that element

Table 1: eZAF Smart Quant Results from Figure 4.

\begin{tabular}{|c|c|c|c|c|c|c|c|c|}
\hline Element & Weight \% & Atomic \% & Net Int. & Error \% & Kratio & Z & A & F \\
\hline C K & 59.31 & 80.92 & 1034.71 & 6.35 & 0.3121 & 0.9866 & 0.5334 & 1.0000 \\
\hline O K & 6.96 & 7.12 & 130.36 & 12.87 & 0.0187 & 0.9507 & 0.2831 & 1.0000 \\
\hline $\mathrm{AlK}$ & 8.61 & 5.23 & 499.45 & 4.27 & 0.0592 & 0.858 & 0.7986 & 1.0029 \\
\hline $\mathrm{SiK}$ & 0.04 & 0.02 & 2.44 & 99.99 & 0.0003 & 0.8791 & 0.828 & 1.005 \\
\hline $\mathrm{S} \mathrm{K}$ & 2.78 & 1.42 & 142.88 & 5.76 & 0.0228 & 0.8654 & 0.9385 & 1.0116 \\
\hline $\mathrm{AgL}$ & 7.01 & 1.06 & 150.67 & 7.49 & 0.0512 & 0.6664 & 1.0954 & 1.0019 \\
\hline $\mathrm{FeK}$ & 6.95 & 2.04 & 127.46 & 5.78 & 0.057 & 0.7621 & 1.0075 & 1.068 \\
\hline $\mathrm{NiK}$ & 1.11 & 0.31 & 16.08 & 19.97 & 0.0093 & 0.7733 & 1.0036 & 1.0725 \\
\hline $\mathrm{CuK}$ & 7.23 & 1.86 & 83 & 7.42 & 0.056 & 0.7358 & 1.0042 & 1.0489 \\
\hline
\end{tabular}

\section{Hybridizing CNTH}

Many types of NMP constituents may be integrated into CNT sheet [8]. The metal particle selection is a very important parameter on the CNHT process. The improvement in CNHT properties compared to the pristine CNT sheet strongly depends on the solubility of carbon in the selected metal at high temperatures. measured in the sample divided by the weight of all elements in the sample multiplied by 100 . Whereas, the atomic percentage is the number of atoms of that element, at that weight percentage, divided by the total number of atoms in the sample multiplied by 100. In spectroscopic analysis, the "ZAF correction method" is used for quantitative analysis of the constituent elements. The intensity of the emitted characteristic X-rays is influenced by the atomicnumber effect $(\mathrm{Z})$, the absorption effect $(\mathrm{A})$ and the fluorescence excitation effect (F) [27].
Therefore, binary phase diagrams and ternary phase diagrams of metal carbon systems are helpful for the metal particle selection process. Some metal particles have higher solubility of carbon compared to other metals. The fundamental process of CNT and graphene growth involves the dissolution of carbon in the metal catalyst's liquid phase and the precipitation of crystalline 
carbon in nanostructure form [9]. The solubility of carbon in the selected metal catalyst depends on the synthesis temperature and metal catalyst size. The physical and chemical properties of the synthesized carbon nanostructure can be tailored by altering the type of metal catalyst along with synthesis temperature and metal catalyst size [10-12]. Similarly, the properties of CNHT depend on the metal particle used, synthesis temperature and particle size. Figure 5a shows how the melting temperature of different metal particles reduce with the reduction of the particle diameter. For CNHT, the metal particle selection should refer to the metal-carbon phase diagram to achieve the application specific properties. The advantage of the CNHT synthesis process is multiple types of metal particles can be used to tailor the property of CNHT. Careful selection of metal particles which are soluble in each other's system can result in new properties of the CNHT.

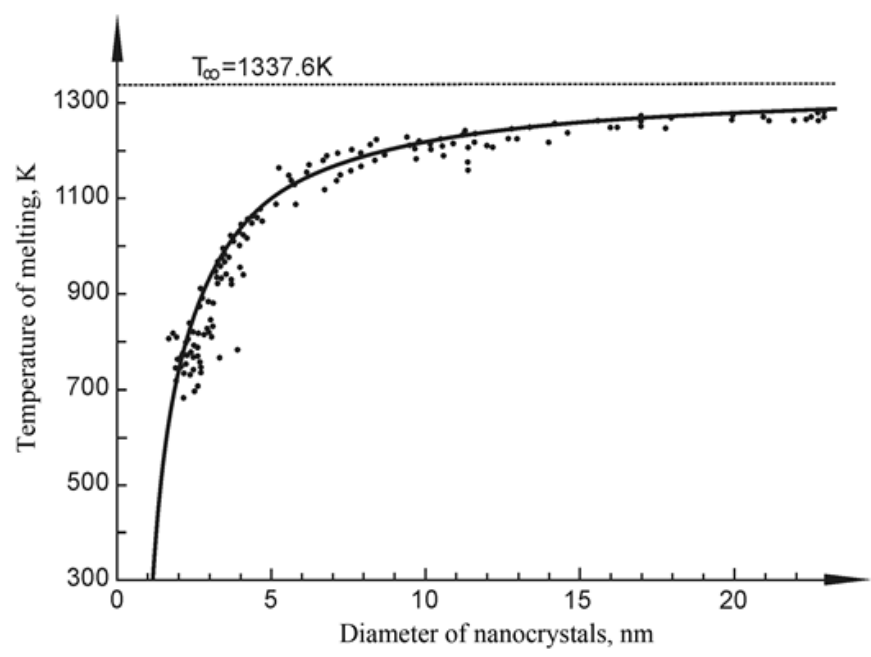

(a)
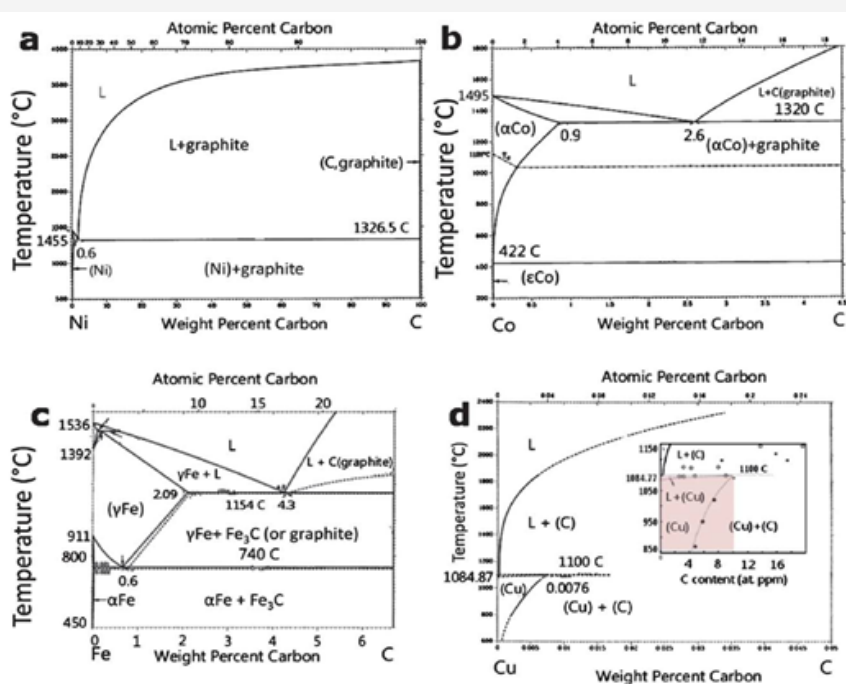

(b)

Figure 5: Synthesizing CNHT: (A) the melting temperature of the metal varies with nanoparticle diameter [9]. (B) the metal must wet carbon or carbon must be partially soluble in the metal, binary phase diagrams of transition metals and carbon, (a) Ni-C; (b) $\mathrm{Co}-\mathrm{C}$; (c) $\mathrm{Fe}-\mathrm{C}$; (d) $\mathrm{Cu}-\mathrm{C}$. Figure reproduced from [13] with permission of The Royal Society of Chemistry. The low carbon solubility in Cu is highlighted in the inset of panel (d).

Generally, there are two classes of particles that can be injected, chemically reacting particles and non-reacting particles. Reacting particles, like transition metals, change shape and may chemically wet or bond to the carbon nanotubes. Non-reacting particles, like carbon or ceramic materials, pass through the reactor and do not interact chemically with the nanotubes. Lower melting temperature particles will melt and conform to the CNT and partly coat the bundles of CNTs. A variety of different NMP in a mixture can be incorporated into the process at once. The amount of NMP used is optimized to achieve the desired properties. Tuning the process is difficult, and only a narrow window of parameters will allow the process to proceed. This condition is being investigated and depends on the type and size of the particle. Combinations of metal particles can be used to form hybrid materials with properties and cost to meet desired applications $[13,14]$. CNT and NMP materials potentially can be composited with functional fibers and advanced materials depending on where they are integrated into the process $[15,16]$.

Increasing the yield of the CNTH process is being investigated considering the capability to inject particles at different points along the reactor tube and by moving away from the use of tubetype reactors.

\section{Safety of Carbon Nanotube Fabric with Nanoparticles}

Nanotube Sheet must be safe for use and must be tested for particle release. The high rate of manufacturing must also be achieved to reduce the cost of the material. These aspects are discussed next.
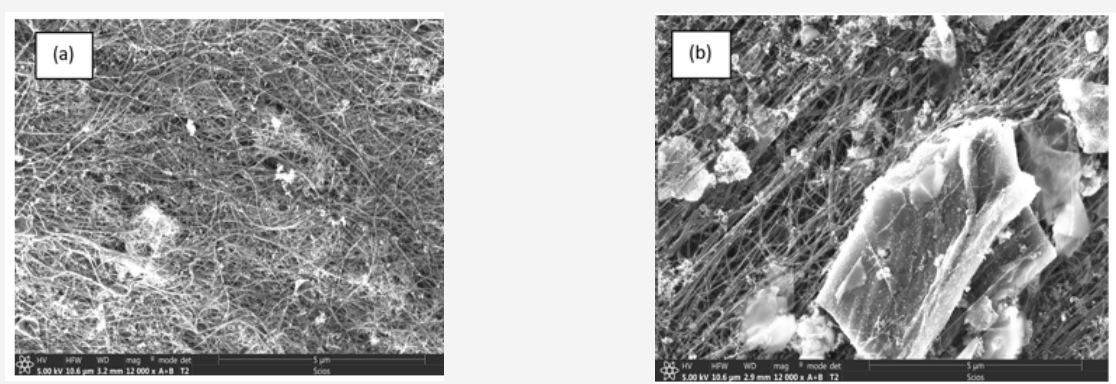

Figure 6: Samples tested for particle release: (a) pristine CNT sheet with residual catalyst particles; (b) CNTH sheet with carbon charcoal particles in the sheet. 
Nanotechnology has brought advancements in many areas such as electronics, photonics, energy, and medicine [14-21]. However, unwanted nanoparticle release during usage or production of these particles is a concern due to the potential impact of nanoparticles on the environment and human health. Therefore, early detection of unwanted nanoparticles in the environment can lead to safer use of nanotechnology [22]. Manufacturing of nanoparticles especially during the cleaning and disposal processes may lead to unwanted human exposure to nanoparticles. Relaxation times (the time taken for a particle released into the flow to move with the velocity of its surrounding flow) for nanoparticles in the nanometer size range are extremely small [23-24]. Traditional detection techniques, such as based on inertia, are only effective at very high velocities [25]. Knowledge of the concentration of nanoparticles in the environment during manufacturing may help to take safety steps [26] to reduce exposure to nanoparticles. The safety of nanotube sheet pristine and with nanoparticles inside must be established in order for the material to be accepted in broad applications. Thus, testing for release of particles from the sheet was performed. Two CNT sheets (sheet 1 pristine, and sheet 2 with microscale carbon particles integrated within the sheet during the nanotube synthesis process) were tested for particle release, Figure 6.

\section{Testing for nanoparticle release from nanotube sheet}

In this test, potential airborne nanomaterials that may have been released from the surface of the CNT sheet are monitored using a personal ultrafine particle (UFP) counter (Figure 7a). For the test, two types of CNT sheets are rubbed/flexed using hands in a glove box to monitor the release of nanomaterials, Figure 7b. Before rubbing/flexing the CNT sheets, the background UFP concentration was measured inside the glove box (which was at the typical level of particle concentration for a room). Subsequently, the UFP concentration was measured during rubbing/flexing of the sheet and the particle level compared to the background concentration.

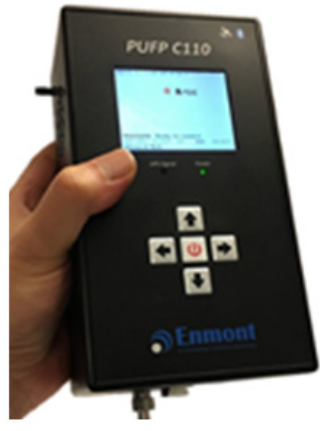

(a)

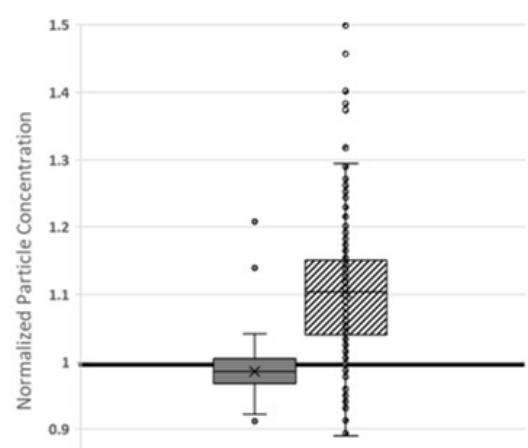

(b)

Figure 7: Testing the release of nanoparticles from nanotube sheet: (a) The PUFP C110 Enmont UFP counter used in this test; (b) The normalized particle concentration (real-time nanomaterial concentration divided by the average background concentration). Nanotube sheet 1 is a pristine sheet. Flexing the sheet did not release nanoparticles (nanotubes or iron catalyst). Nanotube sheet 2 is a sheet with micron carbon particles integrated into and on the sheet. Flexing the sheet released particles. Since the carbon particles do not react with the CNT, they are not bound to the surface of the CNT sheet and can be released which is expected. Thus, nanotube face sheets or functional grading should be used to prevent the release of particles when non-reactive particles are used.

\section{Instrument for particle counting}

The current wearable Enmont LLC PUFP counters are relatively small, lightweight and rugged. The Enmont PUFP counters are water-based condensation particle counters (CPC) that measure particle concentrations down to the size of 5 nanometers in aerodynamic diameter in near real-time ( $300 \mathrm{msec}$ response time). Also, the Enmont PUFP counters are able to provide ongoing single particle count measurement from any geometrical position and sustained mobile conditions ( \pm 4 gravitational acceleration). The Enmont PUFP counters maintain pinpoint accuracy even when they are turned upside down or jostled, therefore, are easy to operate.

\section{Results}

Figure $7 \mathrm{~b}$ shows the normalized particle concentration during rubbing/flexing. The normalized particle concentration is the realtime particle concentration measured near the CNT sheet divided by the average background concentration of nanoparticles. The measurements were performed from two CNT sheets (sheet 1 pristine, and sheet 2 with micron carbon particles integrated within the sheet during the nanotube synthesis process). No cleaning or post-processing of the sheets was performed. The NMP in the CNT sheet 1 are iron catalyst particles and are adhered to the CNTs. The results show that no noticeable nanomaterial release from sheet 1 was detected. In addition to the catalyst particles that come from the synthesis reaction, carbon nanoparticles are injected on the second CNT sheet. For sheet 2, it is observed that the rubbing action released particles which were detected by the instrument. This initial testing indicates that non-reactive particles like carbon can be released from the surface of the sheet. Thus, a pristine cover sheet may be needed to prevent particle release. Alternatively, acid treatment or rolling post-processing may be needed to prevent the release of the particles. In some applications, the release of particles may be part of the functional design of the fabric or functional textile material. The nanotubes are mm length and entangled and are not released from the fabric.

\section{Functionally graded textiles}

Functionally graded CNT sheet, Figure 8, is being investigated for safety and performance for various applications. To accomplish this, the Functional Nanoparticle (FNP) injectors used in the synthesis process must inject particles without agglomeration at 
low flow rates of the carrier gases. Integrating different types of NMP into the reaction and characterizing the material produced is being performed. Grading the amount of nanoparticles throughout the textile allows the properties to be varied.

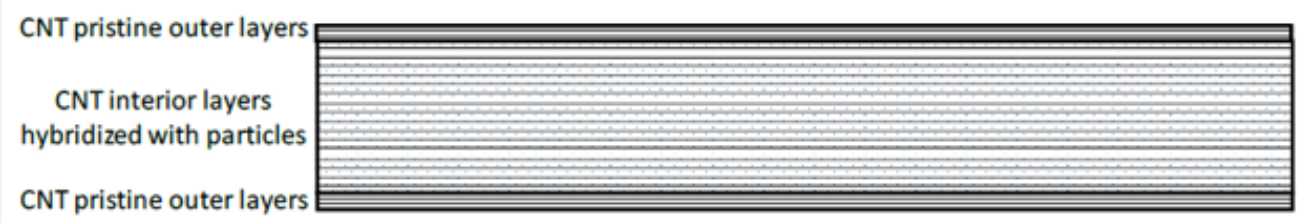

Figure 8: CNHT functional textile. The CNT sheet side view illustrates many layers from hundreds of revolutions wrapping CNT sock onto a drum at 20 RPM for 2-3 hours. The CNT hybrid sheet can be functionally graded with different concentrations of particles for controlling porosity and tunable properties through the thickness of the sheet. Also, the top and bottom layers are pristine to reduce the release of particles from the interior of the sheet.

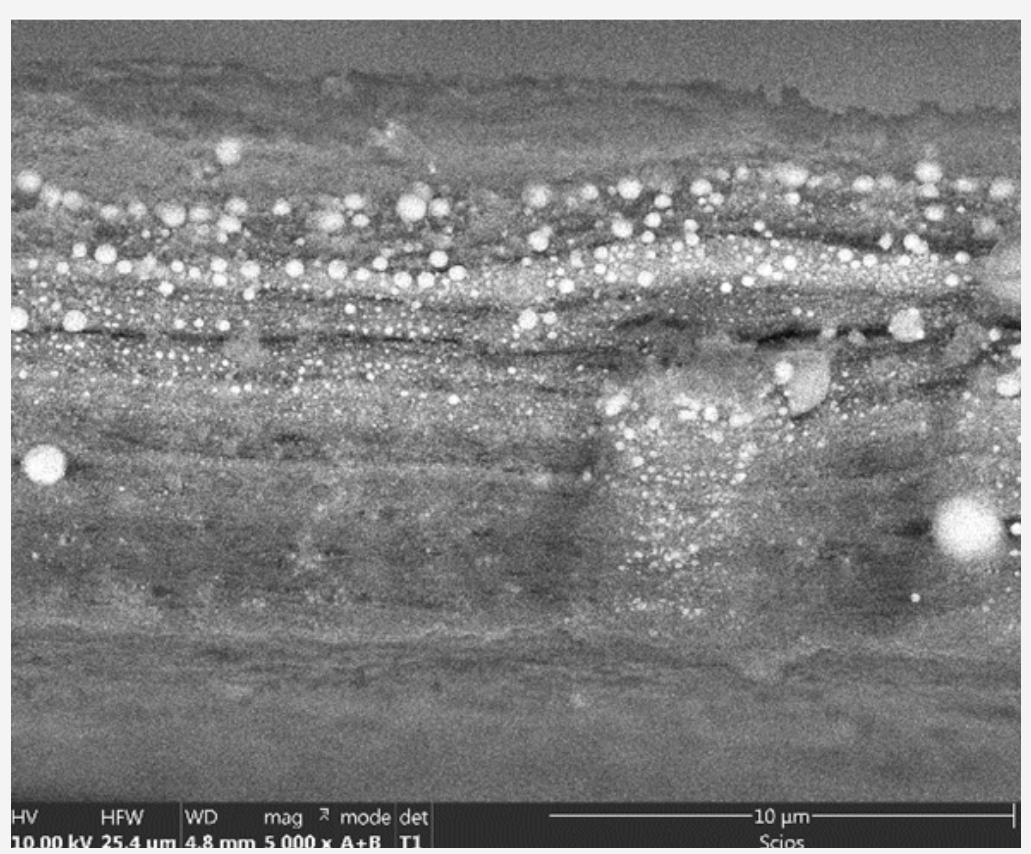

Figure 9: CNHT with Cu NMP. The distribution of $\mathrm{Cu}$ particles is greater at the top and less at the bottom of the figure. The side view of the sheet is shown.

Figure 9 shows functionally grading the CNT sheet with $\mathrm{Cu}$ nanoparticles. Some of the particles agglomerate forming larger particles. The distribution of particles can affect the porosity, conductivity, particle filtering size, and other properties of the sheet.

\section{Summary and Conclusion}

CNTH material is a new development for textiles. The hybridization adds additional properties to CNT sheet and may reduce certain properties depending on the particles used. Potentially many applications can benefit from customization of the properties of CNT sheet. Design of the sheet through nanoparticle selection and functional grading is important to provide the technical performance and safety desired. Carbon nanotube textiles with nanoparticles are achieving properties that will enable their insertion into more and more applications. If low-cost scale-up of the material manufacturing is achieved, the economic impact of nanotube textiles will be significant. The CNHT method is a one-step process with several outstanding advantages for manufacturing customizable carbon nanotube metal and ceramic materials. Carbon nanotube hybrid textiles may become a new common industrial material.

\section{Acknowledgment}

This research was broadly supported by ONR Award N0001415-1-2473; the NSF ERC EEC-0812348; the UCTAC Seed Grant under ESP TECH 15-0160; the Univ. of Cincinnati Education and Research Center Targeted Research Training program (UC ERC-TRT Program); the Water Environment \& Reuse Foundation; and the NSF I/UCRC Center for Intelligent Maintenance Systems (IMS).

\section{Conflict of Interest}

No conflict of interest.

\section{References}

1. Yetisen AK, Qu H, Manbachi A, Butt H, Dokmeci MR, et al. (2016) Nanotechnology in textiles. ACS Nano Rev 10(3): 3042-3068.

2. Schulz MJ, Hou G, Ng V (2018) Patent pending: Methods of manufacturing carbon nanotube (CNT) hybrid sheet and yarn by gas phase assembly, and CNT-hybrid materials. University of Cincinnati. 
3. Capps RC (2011) Carbon nanotube fibers and ribbons produced by a novel wet-spinning process. The University of Texas at Dallas, USA p. 65

4. Bradford PD, Wang X, Zhao H, Maria JP, Jia Q et al. (2010) A novel approach to fabricate high volume fraction nanocomposites with long aligned carbon nanotubes, Composites Science and Technology 70(13): 1980-1985.

5. Liu W, Zhang X, Xu G, Bradford PD, Wang X, et al. (2011) Producing superior composites by winding carbon nanotubes onto a mandrel under a poly (vinyl alcohol) spray. Carbon 49(14): 4786-4791.

6. Motta M, Kinloch I, Windle A (2005) Mechanical properties of continuously spun fibers of carbon nanotubes. Nano Letters 5(8): 15291533.

7. Schulz MJ, Shanov VN, Yin Z (2013) Nanotube superfiber materials. Changing Engineering Design, Elsevier, USA, p. 848.

8. US Research Nanomaterials Inc.

9. Gromov DG, Gavrilov SA (2013) Heterogeneous melting in low-dimensional systems and accompanying surface effects. Thermodynamics - Physical Chemistry of Aqueous Systems InTech.

10. Gohier A, Ewels CP, Minea TM, Djouadi MA (2008) Carbon nanotube growth mechanism switches from tip- to base-growth with decreasing catalyst particle size. Carbon 46(10): 1331-1338.

11. Hou G, Chauhan D, Ng V, Xu C, Yin Z, et al. (2017) Gas phase pyrolysis synthesis of carbon nanotubes at high temperature. Materials and Design 132: 112-118.

12. Peng KJ, Wu CL, Lin YH, Liu YJ, Tsai DP, et al. (2013) Hydrogen-free PECVD growth of few-layer graphene on an ultra-thin nickel film at the threshold dissolution temperature. J Mater Chem C 1(24): 3862.

13. Mattevi C, Kim H, Chhowalla M (2011) A review of chemical vapor deposition of graphene on copper. J Mater Chem 21(10): 3324-3334.

14. Zhang W, Wang C (1997) Synthesizing nanoscale iron particles for rapid and complete dichlorination of TCE and PCBs. Environ Sci Technol 31(7): 2154-2156
15. Cherenach K, Pieterson LV (2012) Smart textiles: Challenges and opportunities. Journal of Applied Physics 112(9): 10.1063/1.4742728.

16.Zhang WX (2003) Nanoscale iron particles for environmental remediation: An overview. J Nanopart Res 5(3-4): 323-332.

17. Biswas $\mathrm{P}, \mathrm{Wu} \mathrm{CY}$ (1998) Control of toxic metal emission from combustors using sorbents: A review. J Air Waste Manag Assoc 48(2): 113-127.

18. Onyango MS, Kojima Y, Matsuda H, Ochieng A (2003) Adsorption kinetics of arsenic removal from groundwater by iron-modified zeolite. J Chem Eng Japan 36(12): 1516-1522.

19. Devarakonda SB, Myers MR, Giridhar D, Dibaji SA, Banerjee RK (2017) Enhanced thermal effect using magnetic nano-particles during highintensity focused ultrasound. PLOS ONE 12(4): e0175093.

20. Lloyd SM, Lave LB, Matthews HS (2005) Life cycle benefits of using nanotechnology to stabilize platinum-group metal particles in automotive catalysts. Environ Sci Technol 39(5): 1384-1392.

21. Peng X, Luan Z, Ding J, Di Z, Li Y, et al. (2005) Ceria nanoparticles supported on carbon nanotubes for the removal of arsenate from water. Mater Lett 59(4): 399-403.

22. Shang J, Gao X (2014) Nanoparticle counting: towards accurate determination of the molar concentration. Chem Soc Rev 43(21): 7267 7278.

23. Masciangioli T, Zhang WX (2003) Environmental technologies at the nanoscale. Environ Sci Technol 37(5): 102A-108A.

24. Colvin V (2003) Point of impact: Where technology collides with society, business, and personal lives. Technol Rev 106: 71-73.

25. Biswas P, Flagan RC (1984) High velocity inertial impactors. Environ Sci Technol 18(8): 611-616.

26. Biswas P, Wu CY (2005) Nanoparticles and the environment. J Air Waste Manag Assoc 55(6): 708-746.

27. MacKay RM. The ZAF model for correction of matrix effects upon measured x-ray intensities. 